\title{
The analysis of BER and SNR based on high altitude platform station in wireless communication network
}

\author{
Xiaoyang Liu ${ }^{1,2}$, Wanping Liu ${ }^{1}$,Chao Liu ${ }^{1}$, Xiaoping Zeng ${ }^{2}$ \\ ${ }^{1}$ School of Computer Science and Engineering, Chongqing University of Technology, \\ Chongqing, 400030, China \\ ${ }^{2}$ Postdoctoral Research Station of Information and Communication Engineering, \\ Chongqing University, Chongqing, 400054, China \\ Email:lxy3103@163.com,www.cqut.edu.cn, zxp@cqu.edu.cn
}

\begin{abstract}
The High Altitude Platform Station (HAPS) wireless communication channel performance of space near network is investigated. The probability density function is derived. The HAPS commutation system model is built. Then, mathematical model under different conditions is evaluated through numerical simulations to verify HAPS commutation system model. The simulation results shows that the proposed method has a better channel performance than the traditional methods. Furthermore, the Bit Error Ratio(BER) has an important influence on the HAPS channel performance.
\end{abstract}

Keywords: High Altitude Platform Station; Channel Performance; Probability Density Function.

\section{Introduction}

HAPS(High Altitude Platform Station),which operates at an altitude about 20 $\mathrm{km}$, is widely known as an alternative mean to deliver both broadband applications and CDMA based IMT-2000 wireless communications using millimeter wave and third generation bands, respectively[1-2].It is an airship equip ping radio transponders which stays in the stratosphere whose weather conditions is comparatively stable for a long period of time. HAPS can be seen as between terrestrial communication system and satellite communication system. HAPS is actually a wireless repeater in the near space. There are well-documented advantages to HAPS communication techniques versus terrestrial communication system. ITU(International Telecommunication Union)described HAPS as the foundation of the next generation of wireless communications, which has the ability to make full use of radio spectrum resources[1-2].

The new traffic model for use in soft and softer handover scheme in HAPS system network with the real mobility management is proposed in Reference[5-7].An analytical model is derived from cell dimensions and the 
user's location. The available bandwidth is managed by the designed control scheme for each ground segment station.

The effect of HAPS instability/movement on the effect to the capacity of CDMA system using two schemes of distance-based power control are studied in reference[8]. ITU has specified that such platforms should be kept within a circle radius sphere of $400 \mathrm{~m}$ with height variations of $\pm 700 \mathrm{~m}$. Spectrum locating and channel positioning are profoundly contingent on mutual interference criterion among services sharing the spectrum. Some techniques such as improved radiation patterns, upsurging minimum elevation angles of the HAPS terminals, DCA (Dynamic Channel Assignment) and environment screening can be applied to facilitate frequency sharing between HAPS and FSS(Fixed Satellite Service).

\section{System Model}

HAPS network includes a set of forward perform routing, and traffic management HAPS communication node. It can be implemented with a laser or microwave communication between them. It is a challenging problem about the link between HAPS.

As can be seen from the Figure 1.HAPS is a highly dynamic space platform. During the communication is related to atmospheric environment, multipath fading, space, flash and other factors. Near space vehicle can stay a long time in the space, usually can stay for several years. The higher the altitude, the better of its field of view. Space-airs safety is the important pillar of national security.

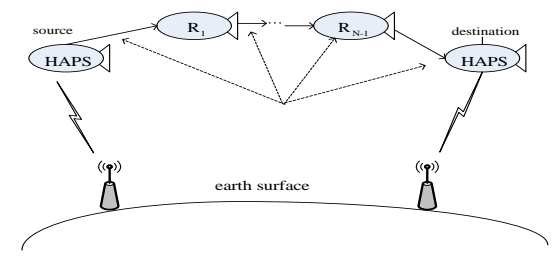

Fig. 1 HAPS space communication network diagram

\section{Mathematical Model}

Every bit of energy in HAPS can be expressed as:

$$
E_{b}=S T_{b}
$$

Where, $S$ is the average power, $T_{b}$ shows the accepted the time required for 1 bit.Signal to noise ratio can be represented as:

$$
\frac{S}{N}=\frac{E_{b} T_{b}}{N_{0} B}=\frac{E_{b} R}{N_{0} B}
$$


In the above formula, $R=1 / T_{b}$ is bit per second, The bit each power and thermal noise power spectral density ratio $E_{b} / N_{0}$ can be repressed as:

$$
\frac{E_{b}}{N_{0}}=\frac{P_{E I R P} g_{A R}}{k T_{s} R l_{F S}}
$$

In the above formula, $P_{\text {EIRP }}$ is the equivalent effective omnidirectional radiation power of the transmitter. ${ }^{g_{A R}}$ is the receiving antenna gain.

As $\gamma_{l}=\bar{\gamma} \alpha_{l}^{2} / N_{t} R_{c}$ can be introduced:

$$
\alpha_{l}=\sqrt{\frac{N_{t} R_{c} \gamma_{l}}{\bar{\gamma}}}
$$

Take a derivative with respect to $\gamma_{l}$.

$$
\frac{\mathrm{d} \alpha_{l}}{\mathrm{~d} \gamma_{l}}=\frac{1}{2} \times\left(\frac{\gamma_{l} N_{t} R_{c}}{\bar{\gamma}}\right)^{-\frac{1}{2}} \frac{N_{t} R_{c}}{\bar{\gamma}}
$$

Then, the PDF(probability density function) of sub-channel's instantaneous signal to noise ratio ${ }^{\gamma_{l}}$ can be repressed as:

$$
f_{\gamma_{l}}(\gamma)=f_{\alpha_{l}}\left(\left(\frac{\gamma_{l} N_{t} R_{c}}{\bar{\gamma}}\right)^{\frac{1}{2}}\right) \times\left|\frac{\mathrm{d} \alpha_{l}}{\mathrm{~d} \gamma_{l}}\right|
$$

$f_{\alpha_{l}}(x)$ is PDF for sub-channel' amplitude $\alpha_{l}$, obey the below formula:

$$
\gamma^{\text {OSTBC }}=\sum_{n=1}^{N_{r}} \sum_{j=1}^{N_{t}} \gamma_{n, j}
$$

Put $f_{\alpha_{l}}(x)$ and $\frac{\mathrm{d} \alpha_{l}}{\mathrm{~d} \gamma_{l}}$ into formula (6). Then, we can get:

$$
\begin{gathered}
f_{\gamma_{l}}(\gamma)=\frac{4 m_{l}{ }^{\left(k_{l}+m_{l}\right) / 2}}{\Gamma\left(m_{l}\right) \Gamma\left(k_{l}\right) \Omega_{l}^{\left(k_{l}+m_{l}\right) / 2}} \frac{\gamma N_{t} R_{c}}{\bar{\gamma}}{ }^{\left(k_{l}+m_{l}-1\right) / 2} \times K_{k_{l}-m_{l}}\left(2\left(\frac{m_{l}}{\Omega_{l}} \times \frac{\gamma N_{t} R_{c}}{\bar{\gamma}}\right)^{1 / 2}\right) \times \frac{1}{2}\left(\frac{\gamma N_{t} R_{c}}{\bar{\gamma}}\right)^{-\frac{1}{2}} \frac{N_{t} R_{c}}{\bar{\gamma}}= \\
\frac{2\left(\frac{m_{l} N_{t} R_{c}}{\Omega_{l} \bar{\gamma}}\right)^{\left(k_{l}+m_{l}\right) / 2}}{\Gamma\left(m_{l}\right) \Gamma\left(k_{l}\right)} \gamma^{\frac{k_{l}+m_{l}-1}{2}} K_{k_{l}-m_{l}}\left(2\left(\frac{\gamma m_{l} N_{t} R_{c}}{\Omega_{l} \bar{\gamma}}\right)^{1 / 2}\right)
\end{gathered}
$$

$\bar{\gamma}_{\text {can be described as: }}$

$$
\bar{\gamma}=\frac{\bar{\gamma}_{l} N_{t} R_{c}}{k_{l} \Omega_{l}}
$$

Put formula (9) into formula (8), ${ }^{f_{l}}(\gamma)$ can be obtained: 


$$
f_{\gamma_{l}}(\gamma)=\frac{2\left(\frac{m_{l} k_{l}}{\gamma_{l}}\right)^{\left(k_{l}+m_{l}\right) / 2}}{\Gamma\left(m_{l}\right) \Gamma\left(k_{l}\right)} \gamma^{\frac{k_{l}+m_{l}}{2} K_{k_{l}-m_{l}}}\left(2\left(\frac{m_{l} k_{l}}{\gamma_{l}} \gamma\right)^{1 / 2}\right)
$$

\section{Simulation Analysis}

The carrier frequency is set to $2 \mathrm{GHz}$. The free space path loss is $130 \mathrm{~dB}$. The influence of atmospheric decline and orthogonal polarization can be negligible. Beam width is $9^{\circ}$. The antenna gain is $33 \mathrm{~dB}$. The antenna is electrically small, has wideband width and good gain characteristics, is efficient and not easily detuned.

In order to reveal the relationship of BER(Bit Error Ratio) and $E_{b} / N_{0}$, the value of estimate and theoretical value are simulated as shown in Figure 2.In Figure 2, the blue line represents the estimate value, and the green line represents the theoretical value.As shown in Figure 2, the estimate value is proportional to the theoretical value.

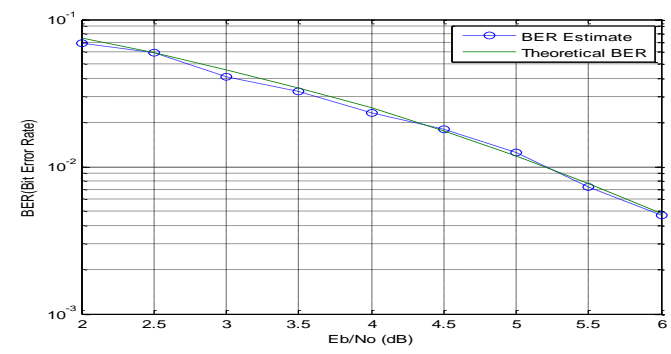

Fig. 2 The relationship of BER and Eb/No

The characteristics of pseudorandom spread spectrum sequence in the BPSK signal exhibit the essential difference between the BPSK signal and other-conventional signals in communications. To trace time-variable HAPS mobile wireless channel, adaptive channel estimators are adopted to provide channel information for MLSE. BPSK(Binary Phase Shift Keying)and MLSE(Maximum Likelihood Sequence Estimation) are researched in the paper. As shown in Figure 3, experimental results show that the proposed method is better than the traditional methods(BPSK,MLSE). 


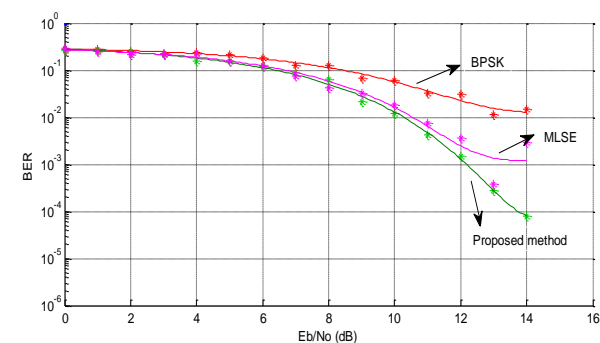

Fig. 3 BER performance analysis

The PDF(probability density function) is suitable for the HAPS channel.The channel attenuation is supposed to $10 \mathrm{~ms}$. As can be seen in Figure 4.The channel attenuation with the blocked situation is the fixed value $-40 \mathrm{~dB}$.

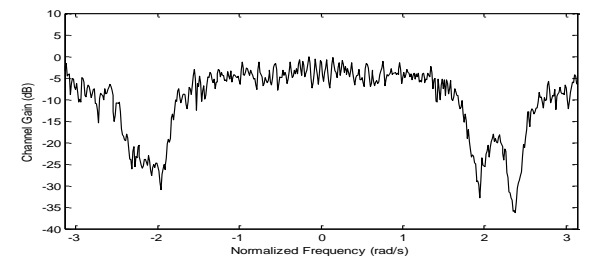

Fig. 4 HAPS channel simulation

As depicted in Figure 5, can observe the S/N(Signal-to-Noise) performance of both channel estimation algorithm when they are deployed for HAPS downlink communication. The transmitting power and receiving antenna gain increased with increasing $\mathrm{S} / \mathrm{N}$.
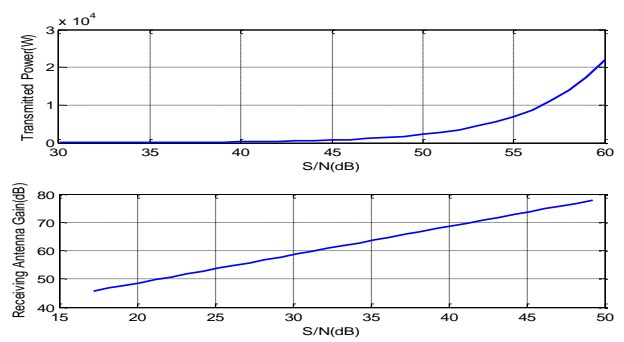

Fig. 5 Signal-to-noise ratio performance

\section{Conclusion}

The HAPS system model is set up, the PDF for HAPC communication channels are derived in this paper.The simulation results show that the proposed method has a better performance than the traditional methods(BPSK,MLSE).It was also shown that the BER has an important influence on the commucation performance. The given expressions can be used to evaluate the channel 
performance of wireless communication networks employed in intelligent highway, and mobile ad hoc applications. In the future, we will consider the impact of multipath fading on the HAPS uplink communication.

\section{Acknowledgments}

The work are supported by Chongqing Postdoctoral Researchers Research Project Special Funded (Xm2015029), Scientific and Technological Research Program of Chongqing Municipal Education Commission (Grant No. KJ1500926, KJ1600923, KJ1500904, KJ1500920), Chongqing Science and Technology Committee Fund of Foundation and Cutting-edge Research Plan(cstc2014jcyjA40007), Research Project of Humanities and Social Sciences of Ministry of Education of China (Grant No. 16YJC860010, 15YJC790061, 16JDSZ2019), Chongqing social sciences planning doctoral project (2015BS059), Natural Science Foundation of China (61571069, 61501065, 61502064, 61503052, 91438104, 11547148).

\section{References}

1. Junil Choi;David J. Love;T. Patrick Bidigare. Coded Distributed Diversity: A Novel Distributed Reception Technique for Wireless Communication Systems[J].IEEE Transactions on Signal Processing,2015, 63(5):1310 -1321 .

2. Israat Tanzeena Haque;Chadi Assi. Profiling-Based Indoor Localization Schemes[J].IEEE Systems Journal,2015, 9(1):76 -85.

3. Hubert Zangl;Mariam Zine-Zine;Stephan Mühlbacher-Karrer. TEDS Extensions Toward Energy Management of Wireless Transducers[J].IEEE Sensors Journal,2015,15(5):587-2594.

4. Jie Hu; Lie-Liang Yang; Lajos Hanzo.Distributed Multistage Cooperative-Social-Multicast-Aided Content Dissemination in Random Mobile Networks[J].IEEE Transactions on Vehicular Technology,2015, 64(7):3075- 3089.

5. UJJINIMATAD R, PARIL $S$ R. Signal detection in cognitive radio networks over AWGN, Nakagami-m and Nakagami-q channels[C]//2014 Eleventh International Wireless and Optical Communications Networks Conference. Vijayawada: IEEE Press, 2014:1-5.

6. BACHA M, HASSAN S A. Distributed versus cluster-based cooperative linear networks: A range extension study in Suzuki fading environments [C]//2013 IEEE 24th Symposium on International Personal Indoor and Mobile Radio Communications.London: IEEE Press, 2013: 976-980.

7. Jing Han; Lingling Zhang; Geert Leus. Partial FFT Demodulation for 
MIMO-OFDM Over Time-Varying Underwater Acoustic Channels[J].IEEE Signal Processing Letters ,2016, 23(2):282-286.

8. Zubair Md. Fadlullah; Tota Nakajo; Hiroki Nishiyama;Yasunori Owada; Kiyoshi Hamaguchi; Nei Kato. Field measurement of an implemented solar powered BS-based wireless mesh network[J].IEEE Wireless Communications,2015,22(3):137-143. 